\title{
Heterodimerization with vascular endothelial growth factor receptor-2 (VEGFR-2) is necessary for VEGFR-3 activity
}

\author{
Antoine Alam ${ }^{\mathrm{a}}$, Jean-Pascal Herault ${ }^{\mathrm{a}}$, Pauline Barron ${ }^{\mathrm{a}}$, Benoit Favier ${ }^{\mathrm{a}}$, Pierre Fons ${ }^{\mathrm{a}}$, \\ Nathalie Delesque-Touchard ${ }^{\mathrm{a}}$, Isabelle Senegas ${ }^{\mathrm{a}}$, Patricia Laboudie ${ }^{\mathrm{a}}$, Jacques Bonnin ${ }^{\mathrm{a}}$, \\ Cecile Cassan $^{\mathrm{a}}$, Pierre Savi ${ }^{\mathrm{a}}$, Bruce Ruggeri ${ }^{\mathrm{b}}$, Peter Carmeliet ${ }^{\mathrm{c}}$, Françoise Bono ${ }^{\mathrm{a}}$, \\ Jean-Marc Herbert ${ }^{\mathrm{a}, *}$ \\ a Sanofi-Synthélabo Research, Cardiovascular Department, Toulouse, France \\ ${ }^{\mathrm{b}}$ Cephalon, Inc, West Chester, PA, United States \\ ${ }^{\mathrm{c}}$ The Center for Transgene Technology and Gene Therapy, Flanders Interuniversity Institute for Biotechnology, KU Leuven, Louvain, Belgium
}

Received 27 August 2004

Available online 2 October 2004

\begin{abstract}
VEGFR-3 is essential for vascular development and maintenance of lymphatic vessel's integrity. Little is known about its cooperative effect with other receptors of the same family. Contrary to VEGFR-2, stimulation of VEGFR-3 by VEGF-C and -D failed to enhance its phosphorylation either in HEK293T or in PAE cells. These ligands were unable to induce angiogenesis of PAEC expressing VEGFR-3 alone. In the presence of VEGFR-2, VEGF-C and -D induced heterodimerization of VEGFR-3 with VEGFR-2. This heterodimerization was associated with enhanced VEGFR-3 phosphorylation and subsequent cellular responses as evidenced by the formation of capillary-like structures in PAE cells and proliferation of primary human endothelial cells expressing both receptors. Taken together, these results show for the first time that VEGFR-3 needs to be associated to VEGFR-2 to induce ligand-dependent cellular responses.
\end{abstract}

(C) 2004 Elsevier Inc. All rights reserved.

Keywords: Angiogenesis; Lymphangiogenesis; CEP-5214; Tyrosine kinase; VEGFR-2; VEGFR-3

Angiogenesis is a crucial component in normal and pathological vascular development [1]. The most important mediators of angiogenesis are members of the vascular endothelial cell growth factor (VEGF) family [2]. The VEGF family of proteins binds to three structurally related receptor tyrosine kinases [3]; known as VEGFR1 which binds VEGF-A, PIGF, and VEGF-B; VEGFR-2 which binds VEGF-A, VEGF-C, VEGF-D, and VEGFE; and VEGFR-3 which binds VEGF-C and VEGF-D. VEGFR-1 and -2 are expressed on vascular endothelial cells, whereas the expression of VEGFR-3 has been

\footnotetext{
* Corresponding author. Fax: +330561 162286.

E-mail address: jean-marc.herbert@sanofi-synthelabo.com (J.-M. Herbert).
}

detected in developing blood vessels [4,5]. In the adult, VEGFR-3 is found on the endothelial cells of lymphatic vessels [5].

In contrast to VEGFR-2, little is known about the signaling and the cellular functions associated with VEGFR3. Even though VEGFR-3 appears to be required for the regulation of the development of the lymphatic system [6], several studies suggest that VEGFR-3 alone is not sufficient to induce the sprouting of lymphatic vessels and appears to require the co-expression of VEGFR-2. Nevertheless, heterodimerization of VEGFR-2 and VEGFR-3 is still a matter of debate. While Pajusola et al. [7] showed that VEGFR-2 is not able to heterodimerize with VEGFR-3 in endothelial cells, it has been shown very recently that the two receptors can associate in response to 
VEGF-C and VEGF-D [8]. Here, we have studied the signaling and the functional consequences on endothelial cell angiogenesis of VEGFR-3. We then showed the fundamental role of VEGFR-2 in the regulation of VEGFR-3 activity.

\section{Materials and methods}

Cells, antisera, and other reagents. Polyclonal antiserum to Flk-1/ KDR (VEGFR-2) was from Santa Cruz Biotechnology (Heidelberg, Germany). Recombinant human VEGF-A and VEGF-D were obtained from R\&D Systems (Lille, France) and VEGF-C was from ReliaTech (Braunschweig, Germany). The horseradish peroxidase (HRP)-conjugated monoclonal antibody anti-phosphotyrosine PY20 was from Zymed, San Francisco, CA. Agarose-conjugated monoclonal anti-HA antibody and the HRP-conjugated anti-HA were from Santa Cruz Biotechnology (Heidelberg, Germany).

Constructs. Human VEGFR-3 deleted in its signal peptide was amplified from pECE-Flt4 (kindly provided by Dr. Birnbaum, INSERM U.119, Marseille) encoding the full length VEGFR-3L, and cloned in pDisplay in-frame with the signal peptide and the HA Tag (Invitrogen). For co-transfection in PAEC, pDisplay-VEGFR-3 was digested with $B a m \mathrm{HI}$ and $N o t \mathrm{I}$, and the VEGFR-3HA product was subcloned into the same sites of pCDNAZeo (Invitrogen). Mutation of the active site of VEGFR-2 was done using the X-L Quick mutagenesis kit (Stratagene) and the following oligonucleotides: ACCGTGGCCGTGCGAATGC TGAAAGAGG and CCTCTTTCAGCATTCGCACAGCCACGGT.

Quantitative real-time PCR. Quantitative RT-PCR assays for human VEGFR-2, VEGFR-3, and TATA-box binding protein (TBP) genes were carried out, in triplicate, using Assays-on-demand Gene Expression Products (PE Applied Biosystems, Weiterstadt, Germany). Real-time PCR conditions were: UNG activation at $50^{\circ} \mathrm{C}$ for $2 \mathrm{~min}$, initial denaturation at $95^{\circ} \mathrm{C}$ for $10 \mathrm{~min}, 40$ cycles, each cycle consisting of denaturation at $95^{\circ} \mathrm{C}$ for $15 \mathrm{~s}$, annealing and extension at $60^{\circ} \mathrm{C}$ for $1 \mathrm{~min}$. Accumulation of fluorescently labeled PCR products was monitored cycle-by-cycle by the GeneAmp 7000 Sequence Detection System (PE Applied Biosystems). Dilutions of known amounts of cloned VEGFR-2, VEGFR-3, and TBP cDNA fragments were used to generate standard curves.

Cell culture and transfection. PAEC (wild-type) and PAEC transfected with VEGFR-2 (PAEC-R2) were cultured in Ham's F-12 medium (Biochrom) containing 10\% fetal calf serum (FCS) and glutamine, at $37{ }^{\circ} \mathrm{C}$ and $5 \% \mathrm{CO}_{2}$. PAEC were transfected using the Lipofectamine method (Gibco/BRL, Carlsbad, CA). HEK293T cells were maintained in DMEM supplemented with 10\% FCS and glutamate. The day before transfection, $3 \times 10^{5}$ cells were seeded on $10-\mathrm{cm}$ Petri dishes and transfection was done using the Fugen 6 (Roche, Indianapolis). HUVECs were electroporated with the HUVEC Nucleofector kit (Amaxa Biosystems). For each transfection, $10^{6}$ cells were combined to $3 \mu \mathrm{g}$ VEGFR-3-plasmid or vector alone. The day after electroporation HUVECs were harvested and used for cell proliferation assay.

Cell proliferation assay. HMVECs (Biowittaker) or HUVECs (Tebu) were seeded $\left(2 \times 10^{3}\right.$ cells/well $)$ in 96 -well plates coated with $0.3 \%$ gelatine. Cells were incubated with saline or VEGF-D at the indicated concentrations. Recombinant proteins were added to the medium every other day. Seven days later, the amount of viable cells was quantified using the cell Titer-glo luminescent cell viability assay (Promega).

Stimulation and cell lysis. Twenty-four hours after transfection, cells were washed and starved overnight in a serum-free medium supplemented with $0.01 \mathrm{mg} / \mathrm{ml} \mathrm{BSA}$. Cells were treated with orthovanadate for $1 \mathrm{~h}$ at $37^{\circ} \mathrm{C}$ and then stimulated or not for $10 \mathrm{~min}$ at $37^{\circ} \mathrm{C}$, with the indicated concentration of VEGF-A, VEGF-C or VEGF-D. After washing with ice-cold PBS containing $100 \mu \mathrm{M} \mathrm{Na} \mathrm{VO}_{4}$, cells were resuspended in lysis buffer containing $150 \mathrm{mM} \mathrm{NaCl}, 20 \mathrm{mM}$ Tris- $\mathrm{HCl}$, pH 7.4, $10 \mathrm{mM}$ EDTA, $1 \%$ Triton $\mathrm{X}-100,100 \mu \mathrm{M} \mathrm{Na} \mathrm{VO}_{4}$, and $1 \mathrm{mM}$ phenylmethylsulfonyl fluoride. The cell lysates were centrifuged at $10,000 \mathrm{~g}$ for $15 \mathrm{~min}$ and the supernatants were collected.

For analysis of VEGFR-2 phosphorylation in PAE cells, stably expressing VEGFR-2 cells were seeded in Petri dishes $\left(10^{6}\right.$ cells per dish) and incubated overnight at $37{ }^{\circ} \mathrm{C}, 5 \% \mathrm{CO}_{2}$. Cells were serumstarved for $2 \mathrm{~h}$ and then incubated with $100 \mathrm{ng} / \mathrm{ml}$ VEGF-A, VEGF-C or VEGF-D for $10 \mathrm{~min}$ at $37^{\circ} \mathrm{C}$. After stimulation, cells were washed with PBS containing $100 \mu \mathrm{M} \mathrm{Na}_{3} \mathrm{VO}_{4}$, lysed, and total protein extracts were subjected to Western blot analysis.

Immunoprecipitation and Western blotting. HA-tagged proteins were immunoprecipitated using an agarose-conjugated anti-HA antibodies. Immune complexes were washed five times with $1 \mathrm{ml}$ lysis buffer. Proteins were eluted by incubation with $50 \mu$ lof $2 \times$ SDS sample buffer and boiling, separated on Novex polyacrylamide gels (Invitrogen), and transferred onto Hybond ECL nitrocellulose membranes (Amersham Pharmacia, Orsay France). Following incubation with 5\% non-fat milk in PBS, the membranes were incubated with: HRP-PY20, 1:1000; HRP-anti-HA,1:5000; or anti-VEGFR-2 (1:1000; Sigma, Lyon, France). Membranes were revealed using ECL detection system and exposure to autoradiography.

In vitro angiogenesis. Growth factor reduced Matrigel (BD Biosciences) was diluted in collagen $(1 / 6 \mathrm{v} / \mathrm{v})$, added to each well of 8 -well culture slides pre-coated with type I rat-tail collagen, and left at $37{ }^{\circ} \mathrm{C}$ for $1 \mathrm{~h}$. Following gel formation, a suspension of cells, mixed or not with VEGFs, was added and incubated at $37{ }^{\circ} \mathrm{C}$ in a humidified $5 \%$ $\mathrm{CO}_{2}$ incubator. After $24 \mathrm{~h}$, cells were rinsed twice with PBS, fixed for 30 min with 4\% PFA, and then stained with Masson's Trichrome stain. The extent of the microcapillary network was measured using an automated computer-assisted image analysis system (Imagenia, Biocom, Les Ulis, France), and the total length of the capillaries in each well was determined. The mean microcapillary network length $(\mu \mathrm{m})$ was calculated for each experimental condition. Experiments were performed in triplicate and repeated three times.

\section{Results}

Angiogenesis of PAEC expressing VEGFR-2 or VEGFR-3

PAEC stably expressing either VEGFR-2 or VEGFR3 were subjected to VEGF-A, -C or -D in the Matrigel model. Morphology of the cells in Matrigel is illustrated in Fig. 1A and the quantification of the total tubules length is shown in Fig. 1B. The results indicate that in PAEC expressing VEGFR-2, VEGF-A induced a potent angiogenic response, whereas VEGF-C had a less pronounced effect and VEGF-D was totally inactive. In VEGFR-3-expressing PAEC, none of the three ligands were able to induce tubule formation (Fig. 1B). These results indicate that VEGFR-3 alone is not sufficient to induce a ligand-dependent angiogenesis in endothelial cells.

\section{VEGF-C-and-D-induced VEGFR-3 phosphorylation}

We then examined the phosphorylation status of VEGFR-3 in response to its ligands in HEK293T cells. The results shown in Fig. 2A indicate that, in the presence of a phosphatase inhibitor (orthovanadate), VEGFR-3 was phosphorylated in a ligand-independent 

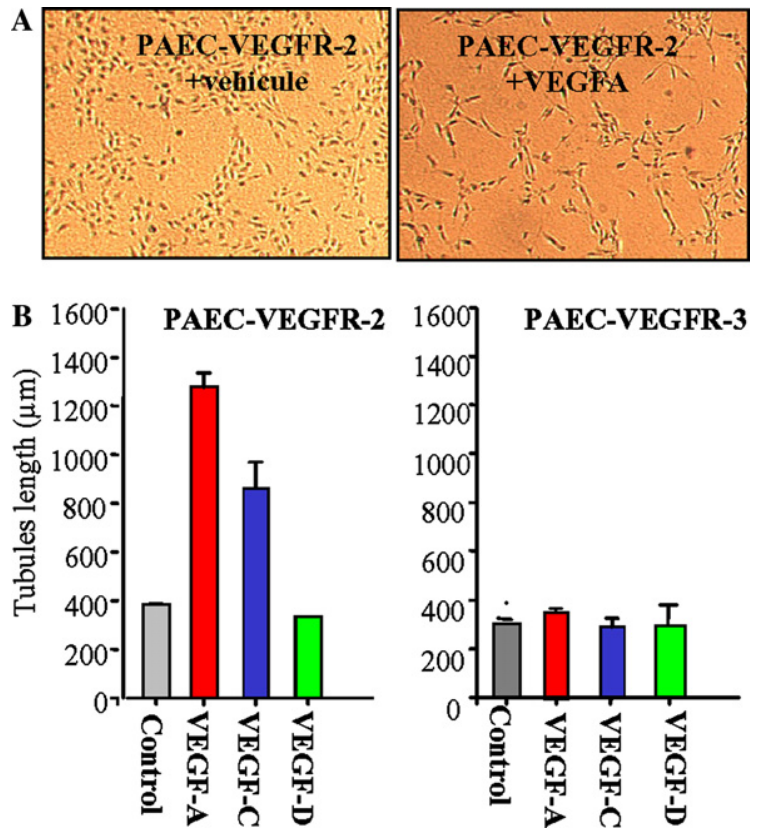

Fig. 1. In vitro angiogenesis of PAEC transfected with VEGFR-2 or VEGFR-3. (A) PAEC-VEGFR-2 (transfected or not) were plated onto the chamber slides and left untreated or stimulated for $24 \mathrm{~h}$ with $100 \mathrm{ng} / \mathrm{ml}$ VEGF-A. Cells were fixed, stained with Masson's Trichrome, and photographed at a 40× magnification. (B) Quantitative analysis of tube length in the PAEC-R2 and PAEC-R3 cells in response to VEGF-A, -C, and -D.
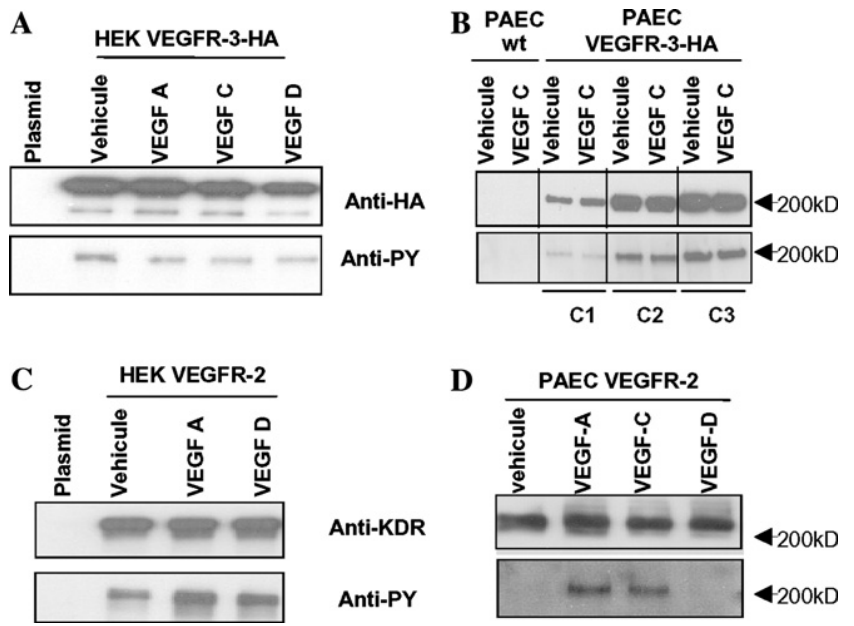

Fig. 2. Effect of VEGFR-2 on the phosphorylation of VEGFR-3. HEK293T cells were transfected with HA-VEGFR-3 (A) or VEGFR-2 (C), treated with orthovanadate $(1 \mathrm{mM})$, and incubated for $10 \mathrm{~min}$ with the vehicle, VEGF-A (10 ng/ml), VEGF-C (100 ng/ml) or VEGF$\mathrm{D}(100 \mathrm{ng} / \mathrm{ml})$. After immunoprecipitation, the receptor was immunoblotted with anti-PY and anti-HA or-VEGFR-2 antibodies. (B) PAEC wt and three clones of PAEC expressing different levels of VEGFR-3 were pre-incubated with orthovanadate and then subjected or not to stimulation with VEGF-C. (D) Phosphorylation of VEGFR2 was assessed as described in Materials and methods.

manner. When the cells were further challenged with different VEGFs (-A, -C or -D), no apparent increase in tyrosine phosphorylation was observed. This result was confirmed in several clones of PAEC expressing different levels of VEGFR-3 (Fig. 2B). Under similar experimental conditions, overexpression of VEGFR-2 in HEK cells was associated to spontaneous phosphorylation of the receptor that was enhanced by VEGF-A and VEGF-D (Fig. 2C). Similarly, in PAEC stably expressing VEGFR-2, in the absence of orthovanadate, the phosphorylation of VEGFR-2 was enhanced by VEGF-A and VEGF-C but not by VEGF-D (Fig. 2D). These latter results were consistent with the results obtained with VEGF-D (Fig. 1B) and with recent observations published by Jia et al. [9] showing that VEGF$\mathrm{D}$, in contrast to VEGF-A, induced a much weaker and slower VEGFR-2 phosphorylation and had little detectable effect on the proliferation of HUVECs. Taken together, these results indicate that neither VEGFR-2 nor VEGFR-3 alone can be activated by VEGF-D.

\section{Interaction of VEGFR-3 with VEGFR-2}

Since some receptor tyrosine kinases such as Tie-1 had to be associated with other tyrosine kinase receptors [10] to induce ligand-dependent cellular signaling, we hypothesized that VEGFR-3 could initiate cell signaling as a result of an interaction with another tyrosine kinase receptor. Since VEGFR-2 has been shown to be always present in VEGFR-3-expressing cells [11-15], we considered VEGFR-2 as a potential regulator of VEGFR-3. In this context, the ability of VEGFR-3 to interact with VEGFR-2 in a ligand-dependent manner was evaluated. HEK293T cells were transfected with HA-VEGFR-3, VEGFR-2 alone or co-transfected with both receptors. Cells were then left untreated or stimulated with VEGF-A or -D. VEGFR-3 was immunoprecipitated and proteins were submitted to Western blot analysis. As shown in Fig. 3A, VEGFR-2 was co-immunoprecipitated with VEGFR-3. Co-immunoprecipitation was increased in cells incubated with VEGF-A or -D. These results show that VEGFR-2 and VEGFR-3 could physically associate in a ligand-dependent manner.

To evaluate the consequences of this ligand-dependent VEGFR-2/R3 association on the phosphorylation of VEGFR-3, the immunoprecipitate was blotted with anti-PY antibodies. The results obtained show that VEGF-A and VEGF-D significantly increased the level of phosphorylation of VEGFR-3 (Fig. 3A), indicating that VEGFR-3 interaction with VEGFR-2 resulted in a ligand-dependent VEGFR-3 phosphorylation.

Interestingly, CEP-5214, a new potent VEGFR- tyrosine kinase inhibitor [16], blocked VEGFR-3 phosphorylation while it did not modify the ligand-dependent heterodimerization (Fig. 3B). These results demonstrate for the first time that heterodimerization of these two receptors is a prerequisite for phosphorylation and signaling through the VEGFR-2/VEGFR-3 complex. Moreover, the signaling through VEGFR-3 required 
$\mathbf{A}$

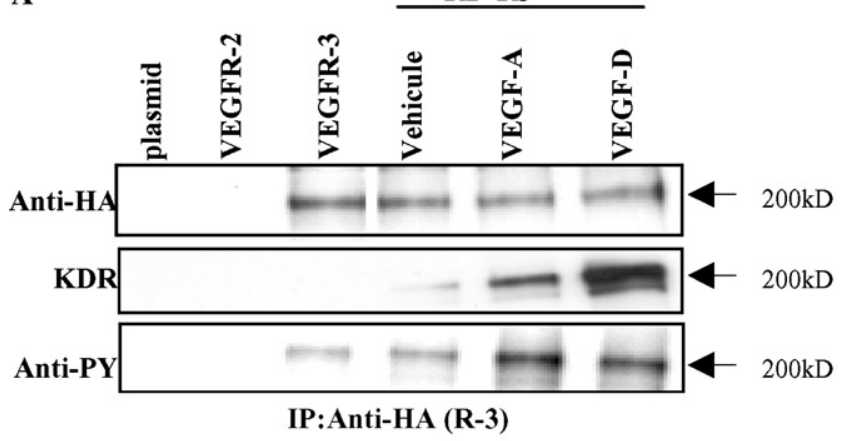

B

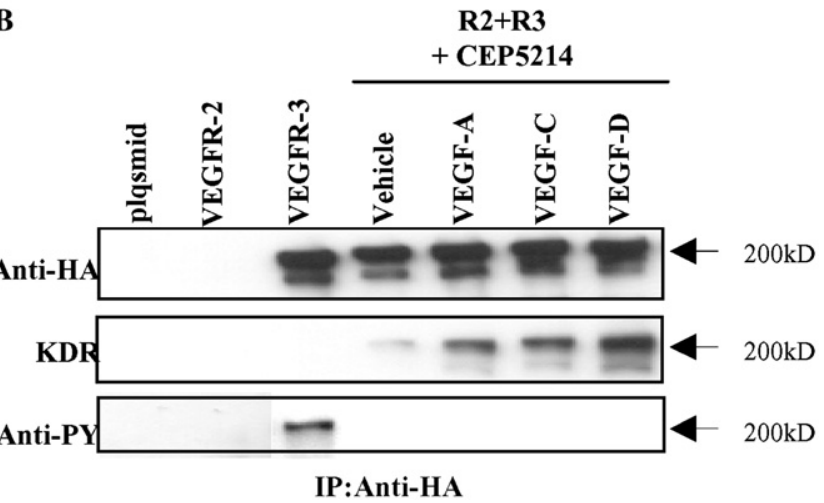

C
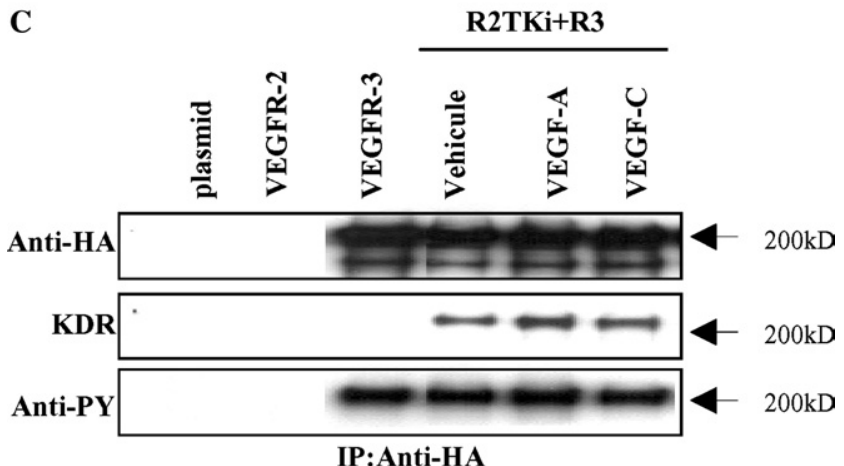

Fig. 3. Association of VEGFR-2 and VEGFR-3. HEK293T cells were transfected with an empty vector (plasmid), HA-VEGFR-3, VEGFR-2 $(\mathrm{A}, \mathrm{B})$ or VEGFR2-Tki $(\mathrm{C})$, or co-transfected with both receptors. The co-transfected cells were challenged as in Fig. 2 except in (C) where $10 \mathrm{nM}$ of CEP-5214 was added to the cells. Cells were then lysed and subjected to immunoprecipitation with an anti-HA antibody. The immunoprecipitated proteins were probed with anti-HA, antiVEGFR-2 or anti-PY antibodies.

heterodimerization with an active VEGFR-2 since the ligand-dependent phosphorylation of VEGFR-3 was abrogated when VEGFR-3 was expressed in the presence of a tyrosine kinase inactive mutant of VEGFR-2 (VEGFR-2-Tki; Fig. 3C).

\section{Angiogenesis of PAEC expressing VEGFR-2 and VEGFR-3}

To assess the physiological significance of the formation of VEGFR-2/VEGFR-3 heterodimers, we evalu-
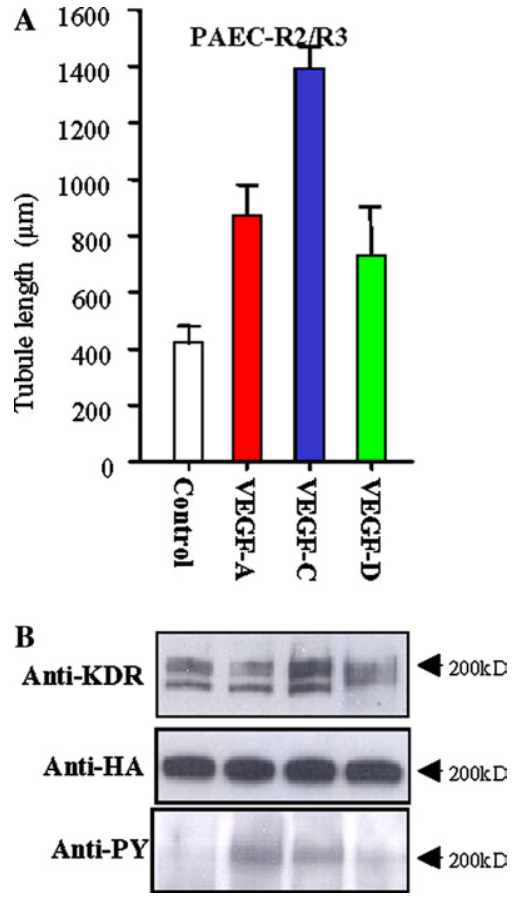

Fig. 4. In vitro angiogenesis of PAEC expressing VEGFR-2 and/or VEGFR-3. (A) Quantitative analysis of tube length in the PAEC-R2/ R3 challenged as in (A). (B) Western blot analysis of VEGFR-2 phosphorylation in response to $100 \mathrm{ng} / \mathrm{ml}$ VEGF-A, VEGF-C, and VEGF-D.

ated whether VEGF-A, -C or -D was able to induce tubule formation in PAEC expressing both receptors. These cells were able to form tubules not only in response to VEGF-A and VEGF-C but also in response to VEGF-D (Fig. 4A). This result confirms that a cooperative interaction between VEGFR-2 and VEGFR-3 is required for VEGF-D-induced cellular responses. Furthermore, VEGF-D was able to induce the phosphorylation of VEGFR-2 (Fig. 4B), showing that VEGFR-3 has to be physically associated to VEGFR-2 to induce the formation of capillary-like tubular structures in response to VEGF-C and/or VEGF-D.

\section{Expression of VEGFR-2 and VEGFR-3 in primary cells}

To further confirm the requirement of both VEGFR2 and VEGFR-3 in response to VEGF-D, we first evaluated by quantitative RT-PCR, the expression levels of VEGFR-2 and -3 mRNA in primary human endothelial cells. The results shown in Fig. 5A indicate that the mRNAs of both receptors are expressed in human microvascular endothelial cells (HMVECs) whereas HUVECs express mostly VEGFR-2. Even though HMVECs expressed two times less VEGFR-2 than HUVECs, proliferation assays indicated that HMVECs responded more efficiently than HUVECs to VEGF-D (Fig. 5B). Ectopic expression of VEGFR-3 in HUVECs induced an enhanced cell proliferation in response to 

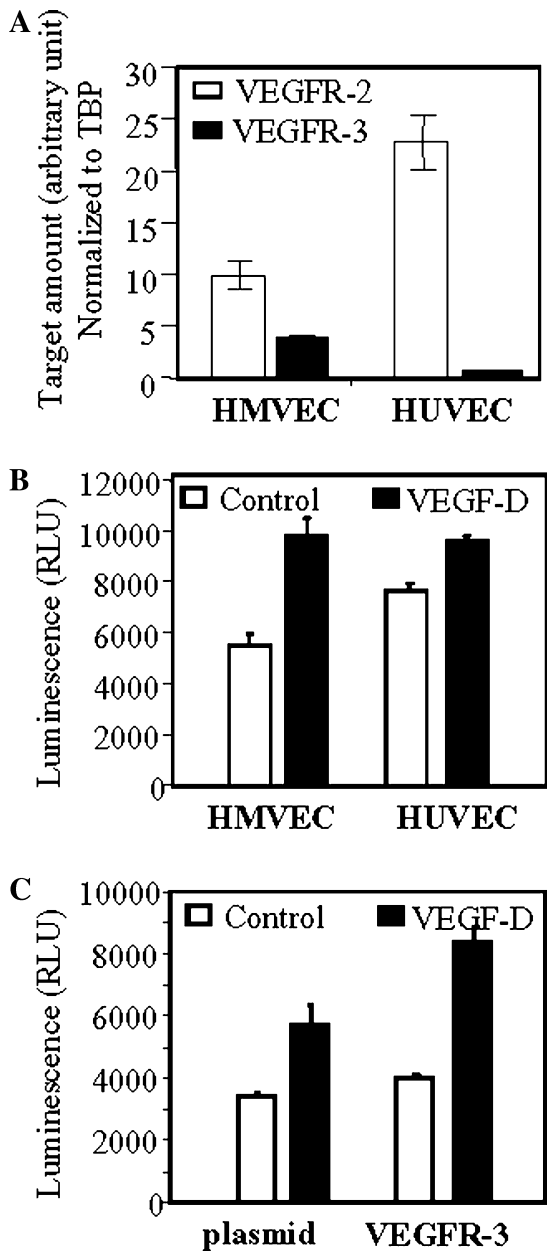

Fig. 5. Proliferation of primary endothelial cells expressing either VEGFR-2 alone or with VEGFR-3, in response to VEGF-D. (A) Real-time quantitative expression of VEGFR-2 and VEGFR-3 mRNA in HMVECs and HUVECs, normalized to TBP mRNA levels. (B) Proliferation of HUVECs and HMVECs in response to vehicle (empty bars) or VEGF-D ( $300 \mathrm{ng} / \mathrm{ml}$; black bars). (C) HUVECs were transfected with an empty vector or with VEGFR-3 (at least fivefold increase in VEGFR-3 mRNA level was routinely obtained). Cells were then subjected to proliferation assay as described in (B). The results shown are means of three independent experiments performed in triplicate.

VEGF-D compared to cells transfected with the empty vector (Fig. 5C). Taken together, these results confirm the requirement of both VEGFR-2 and VEGFR-3 for an efficient response of endothelial cells to VEGF-D.

\section{Discussion}

In this paper, we have demonstrated for the first time that VEGF-C and VEGF-D are unable to enhance the basal level of VEGFR-3 phosphorylation unless it is co-expressed with VEGFR-2. Our results indicate that the VEGFR-3 kinase function is not defective per se but that the receptor alone did not seem to be able to couple to one or several cellular signal transduction mol- ecules. Using "wild-type" VEGFR-3 constructs, phosphorylation of the receptor was undetectable unless the cells were pre-incubated with phosphatase inhibitors such as orthovanadate or $\mathrm{H}_{2} \mathrm{O}_{2}[17,18]$ or after an in vitro activation step following cell lysis [8]. Using a chimeric receptor comprising the extracellular domain of CSF-1R and the VEGFR-3 kinase [7,19,20], CSF-1 has been shown to induce VEGFR-3 phosphorylation, suggesting that the extracellular domain of VEGFR-3 contains structural domains that negatively regulate VEGFR-3 signaling. Finally and more importantly, increase in VEGFR-3 phosphorylation by its ligands was only observed in cells naturally co-expressing VEGFR-2 and VEGFR-3 [11,18,21].

We have demonstrated that VEGFR-3 heterodimerizes with VEGFR-2 in a ligand-dependent manner. CEP-5214, a VEGFR-tyrosine kinase inhibitor, blocked the phosphorylation of VEGFR-3 without affecting its heterodimerization with VEGFR-2, indicating that the signaling cascade of this hetero-complex is similar to that occurring under activation of homodimer tyrosine kinase receptors. Moreover, enhancement of VEGFR3 phosphorylation by its ligands was dependent on the kinase activity of VEGFR-2. These results suggested for the first time that one potential mechanism for initiating a cellular response of VEGFR-3 would be through the creation of phosphotyrosine docking sites on the receptor by transphosphorylation following heterotypic interaction with VEGFR-2. We also showed that PAEC expressing both receptors became sensitive to VEGF-D, which induced VEGFR-2 phosphorylation and tubule formation. The same results were also obtained in a proliferation assay using primary HMVECs expressing VEGFR-2 and VEGFR-3 or with HUVECs after ectopic expression of VEGFR-3. These results are in agreement with the evidence now emerging from a range of studies suggesting that in addition to VEGFR-3, VEGFR-2 may also be required for lymphangiogenesis: Indeed, VEGFR-3 is involved in the regulation of the lymphatic vessel growth and development, since primary human lymph edema has been mapped to missense mutations inactivating the VEGFR-3 kinase [22]. However, signaling through VEGFR-3 does not seem to be sufficient for lymphangiogenesis in vivo. On one hand, chronic delivery of VEGF-C156S (VEGFR-3-specific) or intradermal injection of a VEGF-C156S-encoding adenovirus predominantly induced lymphatic enlargement, on the other hand overexpression of VEGF-C and VEGF-D, which recognize VEGFR-2 as well VEGFR-3, in transgenic mice leads to vessel enlargement, but also lymphatic endothelial cell proliferation and induced lymphatic vessel sprouting during embryogenesis [23-26]. A soluble VEGFR-3-Fc fusion protein, that blocks the interaction of VEGF-C and -D with VEGFR-2 and VEGFR-3, inhibits lymphangiogenesis $[13,27]$. Moreover, VEGFR-2 is expressed in collecting 
lymphatic vessels and in cultured lymphatic endothelial cells $[13,28]$. In light of our observations, these results indicate that VEGF-C and VEGF-D, through an interaction with both VEGFR-3 and VEGFR-2, play an important role in lymphangiogenesis through the formation of VEGFR-3/VEGFR-2 heterodimers.

Finally, we showed that VEGF-A enhanced not only the heterodimerization of VEGFR-3 with VEGFR-2 but also the phosphorylation of VEGFR-3. Similar results were previously described with the EGF/ErB family of receptors. The EGF-like growth factors activate ErbB receptors by promoting receptor-mediated homodimerization or alternatively, by the formation of heterodimers with the orphan ErbB-2 receptor [29]. In addition to the ErB receptor family, a number of studies have demonstrated a regulation of a tyrosine kinase receptor in heterodimeric complexes. It has been shown that heterodimers of VEGFR-1 and -2 may also be formed in VEGF-A-treated cells. The heterodimers formed signal with an efficiency equal to or greater than that of the VEGFR-2 homodimers [30]. Recently, it has also been demonstrated that Tie- 1 and Tie- 2 exist as a pre-formed complex and that Tie-1 induces signaling only by heterodimerization with Tie-2 [10].

Altogether, these results indicate that VEGFR-3 should be added to the growing list of tyrosine kinase receptors that function by heterodimerization with another tyrosine kinase receptor.

\section{References}

[1] W. Risau, Mechanisms of angiogenesis, Nature 386 (1997) 671674.

[2] N. Ferrara, H.P. Gerber, J. Lecouter, The biology of VEGF and its receptors, Nat. Med. 9 (2003) 669-676.

[3] T. Matsumoto, L. Claesson-Welsh, VEGF receptor signal transduction, Sci. STKE re21 (2001) 1-17.

[4] A. Kaipainen, J. Korhonen, T. Mustonen, V.W. van Hinsbergh, G.H. Fang, D. Dumont, M. Breitman, K. Alitalo, Expression of the fms-like tyrosine kinase 4 gene becomes restricted to lymphatic endothelium during development, Proc. Natl. Acad. Sci. USA 92 (1995) 3566-3570.

[5] E. Kukk, A. Lymboussaki, S. Taira, A. Kaipainen, M. Jeltsch, V. Joukov, K. Alitalo, VEGF-C receptor binding and pattern of expression with VEGFR-3 suggests a role in lymphatic vascular development, Development 122 (1996) 3829-3837.

[6] H. Kubo, T. Fujiwara, L. Jussila, Y. Sakai, K. Alitalo, Y. Yamaoka, S.I. Nishikawa, Involvement of vascular endothelial growth factor receptor-3 in maintenance of integrity of endothelial cell lining during tumor angiogenesis, Blood 96 (2000) 546-553.

[7] K. Pajusola, O. Aprelikova, G. Pelicci, H. Weich, L. ClaessonWelsh, K. Alitalo, Signalling properties of FLT4, a proteolytically processed receptor tyrosine kinase related to two VEGF receptors, Oncogene 9 (1994) 3545-3555.

[8] J. Dixelius, T. Mäkinen, M. Wirzenius, M. Karkkainen, C. Wernstedt, K. Alitalo, L. Claesson-Welsh, Ligand-induced vascular endothelial growth factor receptor-3 (VEGFR-3) heterodimerization with VEGFR-2 in primary lymphatic endothelial cells regulates tyrosine phosphorylation sites, J. Biol. Chem. 278 (2003) 40973-40979.
[9] H. Jia, A. Bagherzadeh, R. Bicknell, M.R. Duchen, D. Liu, I. Zachary, Vascular endothelial growth factor (VEGF)-D and VEGF-A differentially regulate KDR-mediated signaling and biological function in vascular endothelial cells, J. Biol. Chem. 279 (2004) 36148-36157.

[10] M.B. Marron, D.P. Hughes, M.D. Edge, C.L. Forder, N.P. Brindle, Evidence for heterotypic interaction between the receptor tyrosine kinases TIE-1 and TIE-2, J. Biol. Chem. 277 (2002) 31768-31773.

[11] T. Veikkola, L. Jussila, T. Makinen, T. Karpanen, M. Jeltsch, T.V. Petrova, H. Kubo, G. Thurston, D.M. McDonald, M.G. Achen, S.A. Stacker, K. Alitalo, Signaling via vascular endothelial growth factor receptor-3 is sufficient for lymphangiogenesis in transgenic mice, EMBO J. 20 (2001) 1223-1231.

[12] A.N. Witmer, B.C. van Blijswijk, J. Dai, P. Hofman, T.A. Partanen, G.F. Vrensen, R.O. Schlingemann, VEGFR-3 in adult angiogenesis, J. Pathol. 195 (2001) 490-497.

[13] A. Saaristo, T. Veikkola, B. Enholm, M. Hytonen, J. Arola, K. Pajusola, P. Turunen, M. Jeltsch, M.J. Karkkainen, D. Kerjaschki, H. Bueler, S. Yla-Herttuala, K. Alitalo, Adenoviral VEGF-C overexpression induces blood vessel enlargement, tortuosity, and leakiness but no sprouting angiogenesis in the skin or mucous membranes, FASEB J. 16 (2002) 1041-1049.

[14] T. Makinen, T. Veikkola, S. Mustjoki, T. Karpanen, B. Catimel, E.C. Nice, L. Wise, A. Mercer, H. Kowalski, D. Kerjaschki, S.A. Stacker, M.G. Achen, K. Alitalo, Isolated lymphatic endothelial cells transduce growth, survival and migratory signals via the VEGF-C/D receptor VEGFR-3, EMBO J. 20 (2001) 4762-4773.

[15] H.Y. Huang, C.C. Ho, P.H. Huang, S.M. Hsu, Co-expression of VEGF-C and its receptors, VEGFR-2 and VEGFR-3, in endothelial cells of lymphangioma. Implication in autocrine or paracrine regulation of lymphangioma, Lab. Invest. 81 (2001) $1729-1734$.

[16] B. Ruggeri, J. Singh, D. Gingrich, T. Angeles, M. Albom, H. Chang, C. Robinson, K. Hunter, P. Dobrzanski, S. Jones-Bolin, L. Aimone, A. Klein-Szanto, J.M. Herbert, F. Bono, P. Schaeffer, P. Casellas, B. Bourie, R. Pili, J. Isaacs, M. Ator, R. Hudkins, J. Vaught, J. Mallamo, C. Dionne, CEP-7055: a novel, orally active pan inhibitor of vascular endothelial growth factor receptor tyrosine kinases with potent antiangiogenic activity and antitumor efficacy in preclinical models, Cancer Res. 63 (2003) 5978-5991.

[17] J.F. Wang, X.F. Zhang, J.E. Groopman, Stimulation of beta 1 integrin induces tyrosine phosphorylation of vascular endothelial growth factor receptor-3 and modulates cell migration, J. Biol. Chem. 276 (2001) 41950-41957.

[18] J.F. Wang, X.F. Zhang, J.E. Groopman, Activation of vascular endothelial growth factor receptor-3 and its downstream signaling promote cell survival under oxidative stress, J. Biol. Chem. 279 (2004) 27088-27097.

[19] E. Fournier, P. Dubreuil, D. Birnbaum, J.P. Borg, Mutation at tyrosine residue 1337 abrogates ligand-dependent transforming capacity of the FLT4 receptor, Oncogene 11 (1995) 921-931.

[20] E. Fournier, P. Blaikie, O. Rosnet, B. Margolis, D. Birnbaum, J.P. Borg, Mutation at tyrosine residue 1337 abrogates liganddependent transforming capacity of the FLT4 receptor, Oncogene 18 (1999) 507-514.

[21] S. Dias, M. Choy, K. Alitalo, S. Rafii, Vascular endothelial growth factor (VEGF)-C signaling through FLT-4 (VEGFR-3) mediates leukemic cell proliferation, survival, and resistance to chemotherapy, Blood 99 (2002) 2179-2184.

[22] M.J. Karkkainen, R.E. Ferrell, E.C. Lawrence, M.A. Kimak, K.L. Levinson, M.A. McTigue, K. Alitalo, D.N. Finegold, Missense mutations interfere with VEGFR-3 signalling in primary lymphoedema, Nat. Gen. 25 (2000) 153-159.

[23] M. Jeltsch, A. Kaipainen, V. Joukov, M. Lakso, H. Rauvala, M. Swartz, D. Fukumura, R.K. Jain, K. Alitalo, Missense mutations 
interfere with VEGFR-3 signalling in primary lymphoedema, Science 276 (1997) 1423-1425.

[24] S.J. Mandriota, L. Jussila, M. Jeltsch, A. Compagni, D. Baetens, R. Prevo, S. Banerji, J. Huarte, R. Montesano, D.G. Jackson, L. Orci, K. Alitalo, G. Christofori, M.S. Pepper, Vascular endothelial growth factor-C-mediated lymphangiogenesis promotes tumour metastasis, EMBO J. 20 (2001) 672-682.

[25] M. Skobe, T. Hawighorst, D.G. Jackson, R. Prevo, L. Janes, P. Velasco, L. Riccardi, K. Alitalo, K. Claffey, M. Detmar, Induction of tumor lymphangiogenesis by VEGF-C promotes breast cancer metastasis, Nat. Med. 7 (2001) 192-198.

[26] S.A. Stacker, C. Caesar, M.E. Baldwin, G.E. Thornton, R.A. Williams, R. Prevo, D.G. Jackson, S. Nishikawa, H. Kubo,
Achen, VEGF-D promotes the metastatic spread of tumor cells via the lymphatics, Nat. Med. 7 (2001) 186-191.

[27] T. Karpanen, M. Egeblad, M.J. Karkkainen, H. Kubo, S. YläHerttuala, M. Jäättelä, K. Alitalo, Vascular endothelial growth factor $\mathrm{C}$ promotes tumor lymphangiogenesis and intralymphatic tumor growth, Cancer Res. 61 (2001) 1786-1790.

[28] M.S. Pepper, Lymphangiogenesis and tumor metastasis: myth or reality, Clin. Cancer Res. 7 (2001) 462-468.

[29] M.A. Olayioye, R.M. Neve, H.A. Lane, N.E. Hynes, The ErbB signaling network: receptor heterodimerization in development and cancer, EMBO J. 3 (2000) 3159-3167.

[30] K.C. Huang, G.M. Andersson, N. Roomans, L. Ito, L. ClaessonWelsh, Signaling properties of VEGF receptor-1 and -2 homoand heterodimers, Int. J. Biochem. Cell Biol. 33 (2001) 315-324. 